\title{
Configurações
}

Revista de sociologia

\section{Estudantes do ensino superior, praxe académica e satisfação com a vida}

Higher Education Students, Hazing and Satisfaction with Life

Étudiants de l'enseignement supérieur, bizutage et satisfactions dans la vie en général

Suzana Nunes Caldeira, Osvaldo Silva, Áurea Sousa, Maria José D. Martins, Maria Mendes e Susana Pinho Botelho

\section{(2) OpenEdition}

\section{Journals}

\section{Edição electrónica}

URL: http://journals.openedition.org/configuracoes/2865

DOI: 10.4000/configuracoes.2865

ISSN: 2182-7419

\section{Editora}

Centro de Investigação em Ciências Sociais

\section{Edição impressa}

Data de publição: 18 Dezembro 2015

Paginação: 97-112

ISBN: 1646-5075

ISSN: 1646-5075

Refêrencia eletrónica

Suzana Nunes Caldeira, Osvaldo Silva, Áurea Sousa, Maria José D. Martins, Maria Mendes e Susana Pinho Botelho, «Estudantes do ensino superior, praxe académica e satisfação com a vida »,

Configurações [Online], 16 | 2015, posto online no dia 29 dezembro 2015, consultado o 01 maio 2019 URL : http://journals.openedition.org/configuracoes/2865; DOI : 10.4000/configuracoes.2865 
Caldeira, Suzana Nunes; Silva, Osvaldo; Sousa, Áurea; Martins, Maria José D.; Mendes, Maria; Botelho, Susana Pinho - Estudantes do ensino superior, praxe académica e satisfação com a vida. Configurações, vol. 16, 2015, pp. 97-112

\title{
Estudantes do ensino superior, praxe académica e satisfação com a vida
}

\author{
SUZANA NUNES CALDEIRA* \\ Universidade dos Açores \\ OSVALDO SILVA* \\ Universidade dos Açores \\ ÁUREA SOUSA** \\ Universidade dos Açores \\ MARIA JOSÉ D. MARTINS*** \\ Instituto Politécnico de Portalegre \\ MARIA MENDES**** \\ Universidade dos Açores \\ SUSANA PINHO BOTELHO**** \\ Universidade dos Açores
}

\section{Resumo}

Estudam-se perceções dos estudantes do ensino superior relativamente à existência ou não de violência nas praxes e relacionam-se essas perceções com níveis de satisfação com a vida. A amostra foi constituída por 585 estudantes de duas instituições do ensino superior português e os dados recolhidos através de um processo de inquérito por questionário. Globalmente, os resultados indicam que entre $10 \%$ e $11 \%$ dos estudantes apontam as praxes como contendo alguma violência. Indicam, ainda, que os níveis de satisfação com a vida apresentam correlação negativa, mas muito baixa, com a perceção dos estudantes sobre a violência nas praxes.

Palavras-chave: ensino superior, praxe académica, violência, satisfação com a vida.

\footnotetext{
* Doutorados, Universidade dos Açores, Centro Interdisciplinar de Ciências Sociais - CICS.UAc/CICS. NOVA.UAc. Email: suzana.n.caldeira@uac.pt I osvaldo.dl.silva@uac.pt.

** Doutorada, Universidade dos Açores, CEEAplA. Email: aurea.st.sousa@uac.pt.

*** Doutorada, Instituto Politécnico de Portalegre; UIDEF - IEUL; CIEP, Portugal. Email: mariajmartins@esep.pt.

***** Licenciadas, Universidade dos Açores. Email: macmendes1@hotmail.com I susanapinhobotelho@ hotmail.com.
} 


\begin{abstract}
Higher Education Students, Hazing and Satisfaction with Life

This study is focused on how students from higher education perceive hazing practices (as violent/non-violent practices) and the relationship between hazing and the levels of satisfaction with life. The sample was comprised by 585 students from two Portuguese higher education institutions and the data was collect through a questionnaire. The results show that $10 \%-11 \%$ of the students describe hazing as an occurrence with some violence. Overall, the results show that the levels of satisfaction with life are related in a negative, but very low, way with the perceptions of violence at hazing practices.
\end{abstract}

Keywords: higher education, hazing, violence, satisfaction with life.

\title{
Résumé
}

Étudiants de l'enseignement supérieur, bizutage et satisfactions dans la vie en général Cette étude vise à analyser les perceptions des étudiants de l'enseignement supérieur concernant l'existence de la violence dans le bizutage ainsi que la relation de ces perceptions avec les niveaux de satisfaction à l'égard de la vie. Nous avons travaillé avec 585 étudiants de deux établissements d'enseignement supérieur au Portugal. Les données ont été collectées sur base d'un questionnaire. Les résultats de l'étude démontrent que 10 à $11 \%$ des étudiants affirment que le bizutage est caractérisé par une certaine violence. Les résultats de l'étude démontrent, de même, une relation négative, mais très faible, entre les niveaux de satisfaction à l'égard de la vie et la perception des étudiants à propos de la violence dans le bizutage.

Mots-clés: enseignement supérieur, bizutage, violence, satisfaction avec la vie.

\section{Introdução}

Nos dias que correm o termo "violência interpessoal" parece já ser de uso comum. Se, por um lado, esta naturalização do uso do termo pode corresponder a uma maior desocultação de toda a problemática da violência praticada por pessoas contra outras pessoas, também pode significar a existência de uma maior proliferação de casos violentos.

Este problema, cujas manifestações vão ressurgindo sob novas formas e roupagens, bem como atingindo indivíduos de diferentes gerações, grupos sociais e contextos ambientais, tem vindo a ser alvo de atenção por parte de investigadores (e.g., Cocco e Lopes, 2010; Mendes, Duarte, Araújo e Lopes, 2013) e de organismos internacionais e nacionais. Os primeiros têm procurado conhecer e caracterizar o fenómeno, no domínio do abuso físico, psicológico ou emocional, sexual, financeiro e, ainda, enquanto resultado de negligência intencional ou não intencional (WHO, 2002), praticado sobre seres humanos mais vulneráveis, como crianças, adolescentes, mulheres e/ou idosos (Zanatta, 2013), em cenários diversos, como escolas, famílias, serviços de saúde, lares residenciais ou centros de dia (Mysyuk, Westendorpa e Lindenberg, 2013; 
Ruotti, 2010), sobretudo com vista a facilitar, às vítimas, uma atitude de proteção e/ou delação da violência. Os segundos têm afirmado a necessidade prioritária da prevenção, tanto em termos de prevenção primária da violência, como em termos de prevenção secundária e terciária (V Plano Nacional de Prevenção e Combate à Violência Doméstica e de Género, 2014-2017; WHO, 2002).

Um dos contextos mais descrito na literatura em termos de ocorrência de diferentes formas de violência é o escolar (Martins, 2007; Ruotti, 2010). Essas vivências, diretas ou vicariantes, de violência fazem perigar, entre outros aspetos, a produção académica dos alunos pelo clima de angústia e medo que se vai instaurando nas organizações educativas. $\mathrm{O}$ relato de situações de agressão física entre alunos e entre alunos e professores, a destruição do património, o consumo e tráfico de drogas e até, embora casos isolados, a prática de homicídios (Ferro, 2013) e massacres não configuram elementos encorajadores da adesão à instituição escolar e restringem a tarefa de processamento cognitivo, por a atividade cerebral estar mobilizada para o evitamento de fontes de perigo.

O ambiente universitário começa, também, a ser alvo de atenção no respeitante à violência, essencialmente por via das praxes académicas. Embora haja alguma alusão, na literatura, a praxes de índole solidária voltadas para a comunidade envolvente das instituições de ensino superior (Mascarenhas e col., s/d), as praxes académicas tendem a ser descritas principalmente como ações ou situações hegemónicas que ocorrem entre estudantes experientes e recém-chegados. Estes, atendendo à falta de familiarização com a nova instituição e respetivos reptos académicos, assim como ao provável afastamento de laços sociais que até então serviam de suporte social informal, entre outros fatores, tendem a encontrar-se mais vulneráveis neste período de iniciação e, por isso, mais suscetíveis de serem sujeitos ao exercício de poder e controlo dos estudantes veteranos. Para além disso, "o espaço de socialização nesses primeiros dias é monopolizado pela praxe e são imensos os mecanismos de persuasão para quem tenha alguma reticência em participar" (Cabral e Mineiro, 2015: 16).

Se bem que o ritual praxista possa ser anunciado como um procedimento para acolher e integrar os caloiros, nem sempre as atividades empreendidas parecem poder classificar-se como promotoras do desenvolvimento das pessoas, capazes de contribuir para a curiosidade intelectual, para a sã convivência e para a construção de pertenças identitárias. Muitas vezes tal ritual é traduzido em atividades ultrajantes e violentas como o forçar ao consumo de bebidas alcoólicas ou outras substâncias tóxicas, sujeitar os recém-chegados a agressões diversas ou obrigar a atos sexuais simulados ou reais (Nirth, 2014); ou, num plano mais organizacional, por exemplo a entoação de cânticos ou ladainhas, muitas vezes acompanhados de coreografias burlescas, que colocam em confronto diferentes cursos e estabelecimentos de ensino, sobressaindo uma lógica de competição, com a valorização de uns e a menorização de outros (Cabral e Mineiro, 2015). 
Contudo, os jovens são conscientes de que a violência em geral e na praxe em particular, ao interferir na sua corporeidade e ao modificar o seu modo de pensar e de interagir com o mundo, compromete o bem-estar e a felicidade (Zanatta, 2013). Compromete igualmente o bem-estar e a felicidade das famílias e da sociedade, designadamente quando sucedem incidentes de extrema violência e/ou de particular incúria, como o que retirou a vida a sete jovens da Universidade Lusófona, em 2013 (Diário de Notícias, 15/12/2013).

Em Portugal, começa a haver um conjunto de investigações em torno do tópico "praxe académica", procurando-se descrever os acontecimentos iniciáticos, compreender o modo como os estudantes percecionam esses acontecimentos e questionar a legitimidade de muitas das práticas adotadas (e.g., Dias e Sá, 2013; Pimentel, Mata e Pereira, 2012; Vieira, 2013; Silva, Caldeira, Mendes e Botelho, 2014; Cabral e Mineiro, 2015; Caldeira, Silva, Mendes e Botelho, 2015). Este estudo vem nessa continuidade e procura contribuir para a discussão desta problemática com dados de duas instituições portuguesas de ensino superior. Mais em concreto, configura uma tentativa de relacionar as perceções dos estudantes sobre a existência ou não de violência nas praxes com os seus níveis de satisfação com a vida.

\section{Metodologia}

Os dados são relativos a 585 estudantes, 199 (34\%) do sexo masculino e $386(66 \%)$ do feminino, de duas instituições portuguesas do ensino superior, a Universidade dos Açores (UAc, $n=431,73,7 \%$ ) e a Escola Superior de Enfermagem de Portalegre (ESEP, $n=154,26,3 \%$ ), e foram recolhidos através de um processo de inquérito por questionário (amostragem não probabilística).

O questionário contém variáveis de caracterização da amostra (por exemplo, sexo, instituição, ano frequentado), a Escala de Avaliação das Situações de Bullying nas Praxes do Ensino Superior (EASBPES), de Matos, Jesus, Simões e Nave (2010), a Escala Abreviada de Satisfação com a Vida dos Estudantes (EASVE), de Marques e Ribeiro (2006), e uma questão aberta, onde os estudantes são convidados a escrever dois adjetivos que, na sua perspetiva, melhor traduzam o seu pensamento sobre as praxes.

A EASBPES é um instrumento constituído por 15 itens de autorresposta, numa escala Likert, de 1 -Discordo totalmente (DT) a 5 - Concordo totalmente (CT) e com a posição intermédia 3 - Não concordo nem discordo (NCND). Neste estudo tomam-se para análise apenas os itens 11 "As praxes continham alguma violência" e 12 "Eu fui agredido(a) por atos ou palavras", os quais são de leitura invertida.

A EASVE é constituída por sete itens de autorresposta, apresentados na Tabela 1, numa escala tipo Likert de 1 - Discordo totalmente (DT) a 6 - Concordo totalmente (CT), com as posições intermédias definidas por 3 
Tabela 1.

Descrição dos itens da EASVE

Itens
V1 A minha vida está a correr bem
V2 A minha vida é perfeita
V3 Eu gostaria de mudar muitas coisas na minha vida
V4 Eu desejava ter uma vida diferente
V5 Eu tenho uma vida boa
V6 Eu tenho na vida o que quero
V7 A minha vida é melhor do que a vida da maioria das outras pessoas da minha idade

- Discordo Pouco (DP) e 4 - Concordo Pouco (CP). Nesta escala os itens 3 e 4 são de leitura invertida.

Os dados foram analisados utilizando diversos métodos estatísticos, de onde se destacam os gráficos paralelos a duas dimensões (2D), a análise de respostas múltiplas, testes de hipóteses não paramétricos (testes de: independência do qui-quadrado, Kolmogorov-Smirnov com a correção de Lilliefors, Mann-Whitney, Kruskal-Wallis, Dunn), a Análise em Componentes Principais Categórica (ACPCat) e a Análise Classificatória Hierárquica Ascendente (ACHA).

As respostas dadas à questão aberta, referente aos adjetivos redigidos pelos estudantes para caracterizar as praxes, foram submetidas a uma análise de respostas múltiplas e a uma análise de conteúdo, da qual resultou a recodificação dessas respostas em três categorias que refletem a opinião dos estudantes sobre as praxes (1 - Negativa (N), 2 - Ambivalente (A), 3 - Positiva (P)). Essa categorização, que originou uma nova variável - "Opinião relativa às praxes" -, foi efetuada por dois juízes independentes, com base no seguinte critério: dois adjetivos de conteúdo/teor desfavorável/negativo (por exemplo, "desnecessárias", "bumilhantes") refletem uma opinião negativa; um adjetivo favorável e outro desfavorável refletem, no seu conjunto, uma opinião ambivalente; dois adjetivos de conteúdo favorável/positivo (por exemplo, "engraçadas", "divertidas”) refletem uma opinião positiva. O acordo interjuízes na categorização dos adjetivos foi de $96,44 \%$.

No respeitante à EASBPES, foi calculada a percentagem de respostas aos dois itens tomados para análise, tendo em conta o grau de acordo expresso pelos respondentes.

Em relação à EASVE, foi calculada a pontuação total (soma das pontuações obtidas nos sete itens) para cada um dos indivíduos, com vista a averiguar a existência ou não de diferenças significativas a nível das pontuações obtidas: 
entre os estudantes dos dois sexos, entre os estudantes das duas instituições, entre os estudantes inscritos nos diversos anos dos cursos, entre os grupos de estudantes definidos pela categorização dos adjetivos, referidos por estes, que refletem a opinião sobre as praxes (1 - negativa, 2 -ambivalente, 3 - positiva).

A ACHA dos itens da EASVE foi efetuada com base no coeficiente de afinidade (Bacelar-Nicolau, 1980, 1988) e em cinco critérios de agregação, dois clássicos (o da ligação simples e o da ligação completa) e três probabilísticos ( $A V L, A V 1$ e $A V B)$, que pertencem a uma família paramétrica de métodos no contexto da metodologia VL (e.g., Nicolau, 1983; Bacelar-Nicolau, 1988; Nicolau e Bacelar-Nicolau, 1998; Lerman, 1972, 1981). A seleção da partição mais significativa ("melhor partição") foi efetuada com base na estatística global de níveis, STAT (e. g., Lerman, 1970, 1981; Bacelar-Nicolau, 1980, 1985).

\section{Apresentação e análise de resultados}

Globalmente, dos 585 alunos, 10,7\% concordaram que as praxes continham alguma violência (afirmação referente ao item 11 da EASBPES). Numa posição de acordo com essa afirmação, encontram-se 13\% dos estudantes da ESEP e $9,9 \%$ dos estudantes da UAc, conforme pode ser verificado pela observação da Figura 1, depreendendo-se, assim, que a percentagem de estudantes de Portalegre que perspetivaram, explicitamente, as praxes como "algo violento"

Figura 1.

Respostas (\%) relativas ao item 11, segundo a instituição.

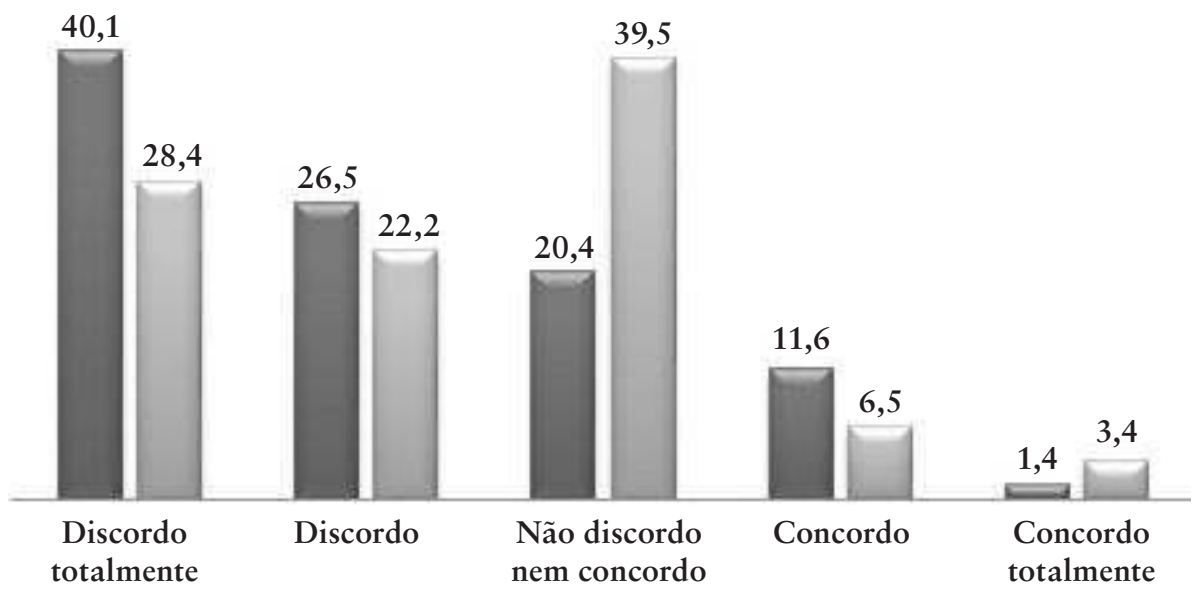

ESEP-IPP

$\mathrm{UAc}$ 
é superior à dos Açores. Constatou-se ainda que 66,6\% dos estudantes da ESEP discordam, pelo menos em parte, com esta afirmação comparativamente a 50,6\% dos estudantes da UAc. É de realçar, ainda, que 20,4\% dos estudantes da ESEP e 39,5\% dos da UAc não manifestaram uma opinião definida neste contexto, já que responderam "Não discordo nem concordo" (polo neutro).

Numa posição concordante com a afirmação associada ao item 12 ("Eu fui agredido(a) por atos ou palavras"), encontram-se $11 \%$ dos 585 inquiridos, sendo de referir que 19,5\% dos estudantes da ESEP e 7,7\% dos estudantes da UAc concordam com essa afirmação, conforme é ilustrado na Figura 2. Assim, no caso dos dados recolhidos, a percentagem de estudantes da ESEP que se sentiu agredida no decurso das praxes é superior à dos da UAc, embora seja de salientar que, também neste caso, a percentagem de estudantes que respondeu "Não discordo nem concordo" foi superior na UAc comparativamente à ESEP (34,1\% versus $27,1 \%)$.

As linhas contínua e a tracejado apresentadas na Figura 3 ligam as categorias mais frequentes das cinco variáveis nela apresentadas, sendo notória a distinção entre as duas linhas no que se refere ao item 11, em que as respostas mais frequentes dos estudantes da UAc se enquadram na categoria "NDNC", enquanto as dos estudantes da ESEP se enquadram maioritariamente na categoria " $D T$ ". Salienta-se ainda que, tanto na UAc como na ESEP, a opinião/ perceção predominante relativamente às praxes foi a de que estas são positivas,

Figura 2.

Respostas $(\%)$ relativas ao item 12 , segundo a instituição.

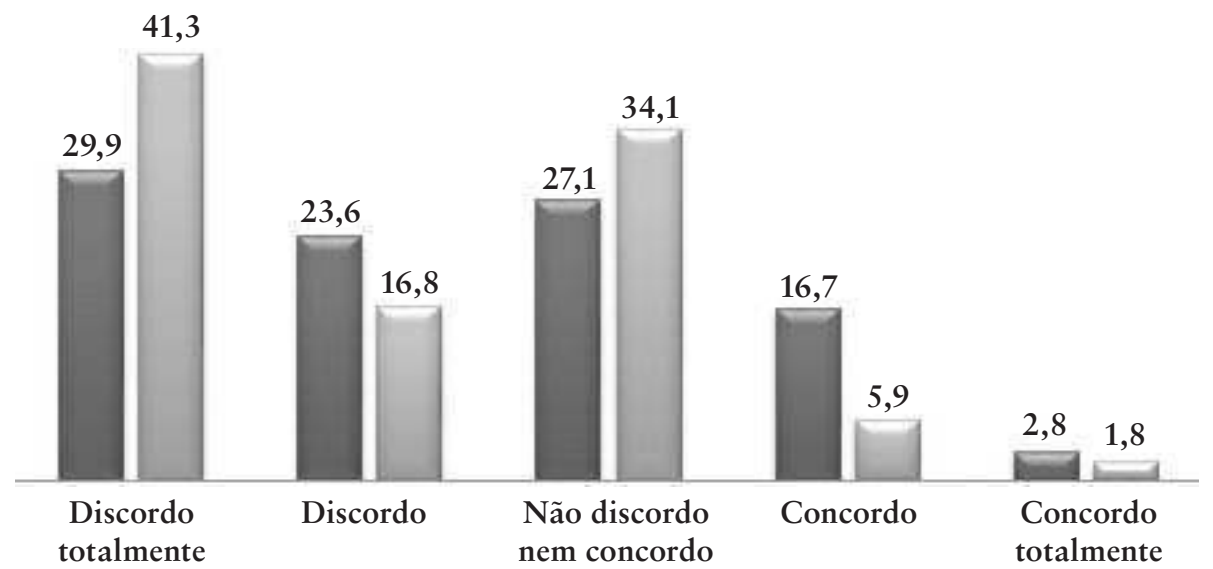

ESEP-IPP

UAc 
Figura 3.

Gráficos Paralelos (2D).

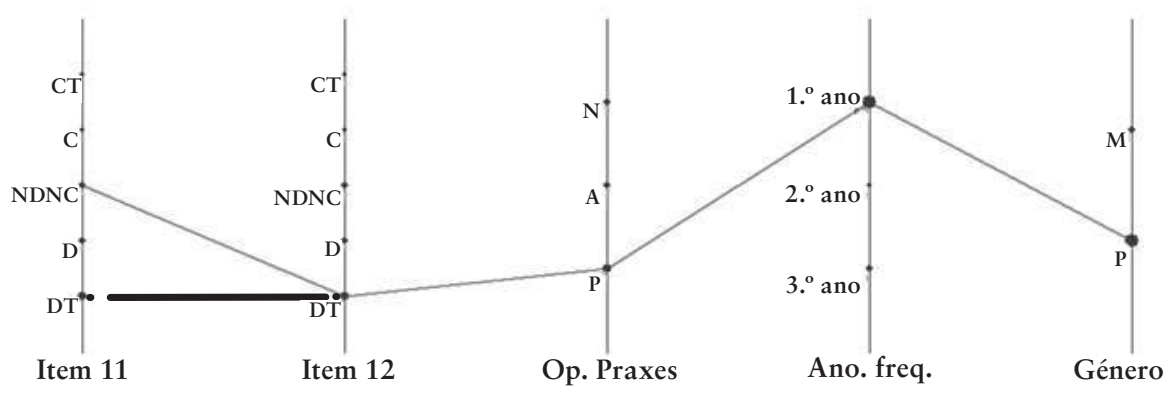

ESEP $\cdots \ldots \ldots \ldots$

UAc

sendo essa a perceção de 51,9\% dos inquiridos da ESEP e de 59,5\% dos da UAc. Esta perceção foi também a de $57,4 \%$ do total dos inquiridos (amostra global), embora $25,1 \%$ dos estudantes tenham uma perceção negativa e $17,4 \%$ uma opinião ambivalente relativamente às praxes.

$\mathrm{O}$ adjetivo mais verbalizado para caracterizar as praxes, "integradoras", foi referido por $35,3 \%$ dos inquiridos e corresponde a $18,4 \%$ do total de respostas. O segundo adjetivo mais verbalizado, referido por $32,8 \%$ dos estudantes, foi "divertidas" e corresponde a $17,1 \%$ das respostas. $\mathrm{O}$ terceiro adjetivo mais verbalizado, "desnecessárias", foi referido por 3,8\% dos estudantes e corresponde a $7,2 \%$ das respostas. Apenas $1,5 \%$ dos estudantes utilizaram o adjetivo "violentas" para descrever as praxes, o qual corresponde a $0,8 \%$ dos diferentes adjetivos listados. Este último foi referido por 2,5\% dos estudantes da ESEP e por 1,1\% dos estudantes da UAc.

A consistência interna dos itens da EASVE, considerando a amostra global, é satisfatória, dado o valor obtido para o coeficiente alfa de Cronbach (0.760). As conclusões foram similares aquando da análise da consistência destes mesmos itens por instituição, tendo-se obtido para o coeficiente alfa de Cronbach os valores de 0.765 e 0.758 , respetivamente, para a ESEP e para a UAc. No que se refere às pontuações totais obtidas na EASVE, a aplicação do teste de KolmogorovSmirnov permitiu-nos rejeitar a hipótese nula de que estas seguem a distribuição normal, pelo que se recorreu a testes não paramétricos. A Tabela 2 apresenta os valores do coeficiente de correlação de Spearman entre: os itens 11 e 12 da $E A S B P E S$; o item 11 e a pontuação total da EASVE; o item 11 e a opinião relativa às praxes; o item $12 \mathrm{e}$ a pontuação total da EASVE; o item $12 \mathrm{e}$ a opinião relativa às praxes; a pontuação da $E A S V E$ e a opinião relativa às praxes. 
Tabela 2.

Valores do coeficiente de correlação de Spearman e de $p$ ( $p$-value) referentes ao respetivo teste de significância.

\begin{tabular}{|l|c|c|c|c|c|c|}
\hline & Amostra & \multicolumn{2}{|c|}{ Género } & \multicolumn{2}{|c|}{ Instituição } \\
\hline & Global & Masculino & Feminino & UAc & ESEP \\
\hline Item 11 vs & $\mathrm{rs}=0.470$ & $\mathrm{rs}=0.497$ & $\mathrm{rs}=0.461$ & $\mathrm{rs}=0.469$ & $\mathrm{rs}=0.516$ \\
item 12 & $\mathrm{p}=0.000$ & $\mathrm{p}=0.000$ & $\mathrm{p}=0.000$ & $\mathrm{p}=0.000$ & $\mathrm{p}=0.000$ \\
\hline Item 11 vs & $\mathrm{rs}=-0.056$ & $\mathrm{rs}=-0.102$ & $\mathrm{rs}=-0.030$ & $\mathrm{rs}=-0.012$ & $\mathrm{rs}=-0.116$ \\
Pontuação da EASVE & $\mathrm{p}=0.200$ & $\mathrm{p}=0.172$ & $\mathrm{p}=0.578$ & $\mathrm{p}=0.823$ & $\mathrm{p}=0.162$ \\
\hline Item 11 vs Opinião & $\mathrm{rs}=0.244$ & $\mathrm{rs}=0.226$ & $\mathrm{rs}=0.250$ & $\mathrm{rs}=0.026$ & $\mathrm{rs}=0.304$ \\
relativa às praxes & $\mathrm{p}=0.000$ & $\mathrm{p}=0.006$ & $\mathrm{p}=0.000$ & $\mathrm{p}=0.000$ & $\mathrm{p}=0.001$ \\
\hline Item 12 vs & $\mathrm{rs}=-0.100$ & $\mathrm{rs}=-0.168$ & $\mathrm{rs}=-0.064$ & $\mathrm{rs}=-0.067$ & $\mathrm{rs}=-0.222$ \\
\hline Pontuação da EASVE & $\mathrm{p}=0.023$ & $\mathrm{p}=0.026$ & $\mathrm{p}=0.234$ & $\mathrm{p}=0.189$ & $\mathrm{p}=0.008$ \\
\hline Item 12 vs Opinião & $\mathrm{rs}=0.211$ & $\mathrm{rs}=0.272$ & $\mathrm{rs}=0.184$ & $\mathrm{rs}=0.191$ & $\mathrm{rs}=0.251$ \\
relativa às praxes & $\mathrm{p}=0.000$ & $\mathrm{p}=0.001$ & $\mathrm{p}=0.002$ & $\mathrm{p}=0.001$ & $\mathrm{p}=0.005$ \\
\hline Pontuação da EASVE vs & $\mathrm{rs}=-0.015$ & $\mathrm{rs}=-0.084$ & $\mathrm{rs}=0.033$ & $\mathrm{rs}=-0.018$ & $\mathrm{rs}=-0.047$ \\
Opinião relativa às praxes & $\mathrm{p}=0.749$ & $\mathrm{p}=0.297$ & $\mathrm{p}=0.562$ & $\mathrm{p}=0.748$ & $\mathrm{p}=0.599$ \\
\hline
\end{tabular}

Concluiu-se que, em geral, os valores do coeficiente de correlação de Spearman foram relativamente baixos, sendo de salientar, como era expectável, a existência de uma correlação positiva significativa $(p \leq 0.01)$ entre os itens 11 e 12, a nível global, nos estudantes do sexo masculino, nos do sexo feminino, nos da UAc e nos da ESEP. É de realçar, ainda, a existência de uma correlação positiva, fraca mas estatisticamente significativa $(p \leq 0.01)$, entre o item 12 e a opinião sobre as praxes. Note-se, finalmente, a existência de correlações negativas muito baixas entre o item 11 e a pontuação total da EASVE, e entre o item 12 e a pontuação total da EASVE.

Com o intuito de testar a existência de diferenças significativas a nível das respostas dadas aos itens 11 e 12 e das pontuações obtidas na EASVE, entre os géneros, entre as instituições e entre os estudantes dos três anos dos cursos do $1 .^{\circ}$ ciclo, foram utilizados os testes de Mann-Whitney e de Kruskal Wallis (testes não paramétricos), cujos valores observados das estatísticas de teste são representados, respetivamente, por $U$ e $H$. No que se refere às pontuações obtidas na $E A S V E$, não se verificaram diferenças significativas: entre os dois géneros ( $U=36000.5$; $p=0.614)$; entre as duas instituições $(U=29778.5$; $p=0.179)$; entre os estudantes inscritos nos diversos anos dos cursos $(H=2.575$; $p=0.276$ ) e entre os grupos de estudantes definidos pelas categorias da variável opinião relativa às praxes $(H=0.236 ; p=0889)$. Emboras as diferenças entre 
as pontuações obtidas na EASVE não sejam estatisticamente significativas, verificou-se que as raparigas apresentaram níveis de satisfação com a vida mais elevados $(M R=289.51)$ comparativamente aos dos rapazes $(M R=282.18)$; os estudantes da ESEP apresentaram pontuações mais elevadas $(M R=302.37)$ comparativamente aos dos da UAc $(M R=281.40)$; os alunos do $3 .^{\circ}$ ano apresentaram pontuações mais elevadas na EASVE $(M R=277.38)$ comparativamente aos do $2 .^{\circ}(M R=271.66)$ e $1 .^{\circ}$ anos $(M R=247.93)$; os estudantes que verbalizaram uma opinião positiva relativa às praxes foram os que apresentaram pontuações mais elevadas na EASVE.

No que respeita às respostas dadas às afirmações associadas ao item 11 , obtiveram-se as seguintes conclusões:

- não se verificaram diferenças estatisticamente significativas entre os dois géneros $(U=30683.5 ; p=0.349)$;

- $\quad$ existem diferenças significativas entre as duas instituições $(U=24293.5$; $p=0.006)$, tendo-se verificado que a média das ordens referente aos alunos da ESEP $(M R=295.74)$ foi maior do que a dos alunos da UAc (MR=256.77). Tal leva-nos a concluir que, embora, conforme já referimos, a percentagem de estudantes da ESEP que perspetivaram, explicitamente, as praxes como "algo violento" seja superior à dos da UAc, os da ESEP tenderam a pontuar mais alto na escala ((1 Concordo totalmente (CT), ..., 5 - Discordo totalmente (DT)), comparativamente aos da UAc, o que indica que no geral tendem a discordar mais com a afirmação referente ao item 11 (recorde-se que 66,6\% dos estudantes da ESEP e 50,6\% dos da UAc discordam, totalmente ou em parte, com a afirmação de que as praxes continham alguma violência);

- existem diferenças significativas entre os estudantes de pelo menos dois dos anos dos cursos do $1 .^{\circ}$ ciclo $(H=8.034 ; p=0.018)$. A aplicação do teste de Dunn permitiu-nos concluir que há diferenças significativas entre os alunos do $1 .^{\circ}$ e $3 .^{\circ}$ anos $(p=0.016)$;

- existem diferenças significativas entre pelo menos dois dos grupos de estudantes definidos pelas categorias da variável opinião relativa às praxes $(1$ - negativa, 2 - ambivalente, 3 - positiva) $(H=32.364$; $p=0.000)$. O teste de Dunn permitiu-nos apurar que as diferenças estatisticamente significativas ocorreram entre os estudantes que consideram as praxes negativas e os que as consideram positivas $(p=0.000)$, assim como entre os que as consideram positivas e os que manifestam uma opinião ambivalente $(p=0.001)$.

No que concerne às respostas dadas às afirmações associadas ao item 12 , obtiveram-se as seguintes conclusões: 
- não se verificaram diferenças estatisticamente significativas entre os dois géneros ( $U=31170 ; p=0.792)$;

- $\quad$ existem diferenças significativas entre as duas instituições $(U=24016$; $\mathrm{p}=0.010$ ), sendo de salientar que os estudantes da ESEP tenderam a pontuar mais baixo $(\mathrm{MR}=239.28)$ na escala $(1-$ Concordo totalmente (CT), ..., 5 - Discordo totalmente (DT)), comparativamente aos da UAc $(M R=275,94)$, o que indica que, comparativamente aos da UAc, os alunos da ESEP tenderam a concordar mais $(19,5 \%$ versus $7,7 \%)$ e a discordar menos $(53,5 \%$ versus $58,1 \%)$ com afirmação de se sentiram agredidos no decurso das praxes (referente ao item 12);

- não existem diferenças significativas entre os estudantes dos três anos dos cursos do $1 .^{\circ}$ ciclo $(H=0.637 ; p=0.727)$;

- existem diferenças significativas entre pelo menos dois dos grupos de estudantes definidos pelas categorias da variável opinião relativa às praxes $(1$ - negativa, 2 - ambivalente, 3 - positiva) $(H=21.634$; $p=0.000)$. O teste de Dunn permitiu-nos apurar que, também neste caso, as diferenças significativas registaram-se entre os estudantes que consideram as praxes negativas e os que as consideram positivas ( $p=0.000)$, e entre os que as consideram positivas e os que têm uma opinião ambivalente relativamente às mesmas $(p=0.035)$.

Segundo o teste de independência do qui-quadrado, existem associações significativas entre: a opinião relativa às praxes e o ano do curso $\left(\chi^{2}=9.565\right.$; $p=0.048)$; o item 11 e a instituição $\left(\chi^{2}=21.880 ; p=0.000\right)$; o item 12 e a instituição $\left(\chi^{2}=21.953 ; p=0.000\right)$. Cerca de $24,4 \%$ dos estudantes do $1 .^{\circ}$ ano, $28,3 \%$ do $2 .^{\circ}$ ano e $18,4 \%$ do $3 .^{\circ}$ ano verbalizaram uma opinião negativa sobre as praxes. Os estudantes dos $1 .^{\circ}(58,6 \%)$ e $3 .^{\circ}$ anos $(71,1 \%)$ têm maioritariamente uma opinião positiva sobre as praxes, enquanto os do $2 .^{\circ}$ ano têm sobretudo uma opinião negativa $(28,3 \%)$ ou ambivalente $(30,4 \%)$.

Foi efetuada a Análise em Componentes Principais Categórica (ACPCat), considerando como variáveis ativas os itens 11,12 e a instituição e como variável suplementar a opinião dos estudantes relativamente às praxes $(1-$ negativa, 2 - ambivalente, 3 - positiva).

A Figura 4 representa o plano que cruza as duas primeiras dimensões, as quais explicam cerca de $99,4 \%(58,3 \%+41,1 \%)$ da variação dos dados. A primeira dimensão opõe, em geral, os estudantes que concordam totalmente com as afirmações relativas aos itens 11 e 12 aos estudantes que discordam totalmente com as afirmações referentes a esses itens. A segunda dimensão opõe os estudantes das duas instituições em estudo. Os itens 11 e 12 estão fortemente associados à dimensão 1. Por outro lado, a variável "Instituição" é 
Figura 4.

Mapa percetual resultante da ACPCat (Variable Principal Normalization)

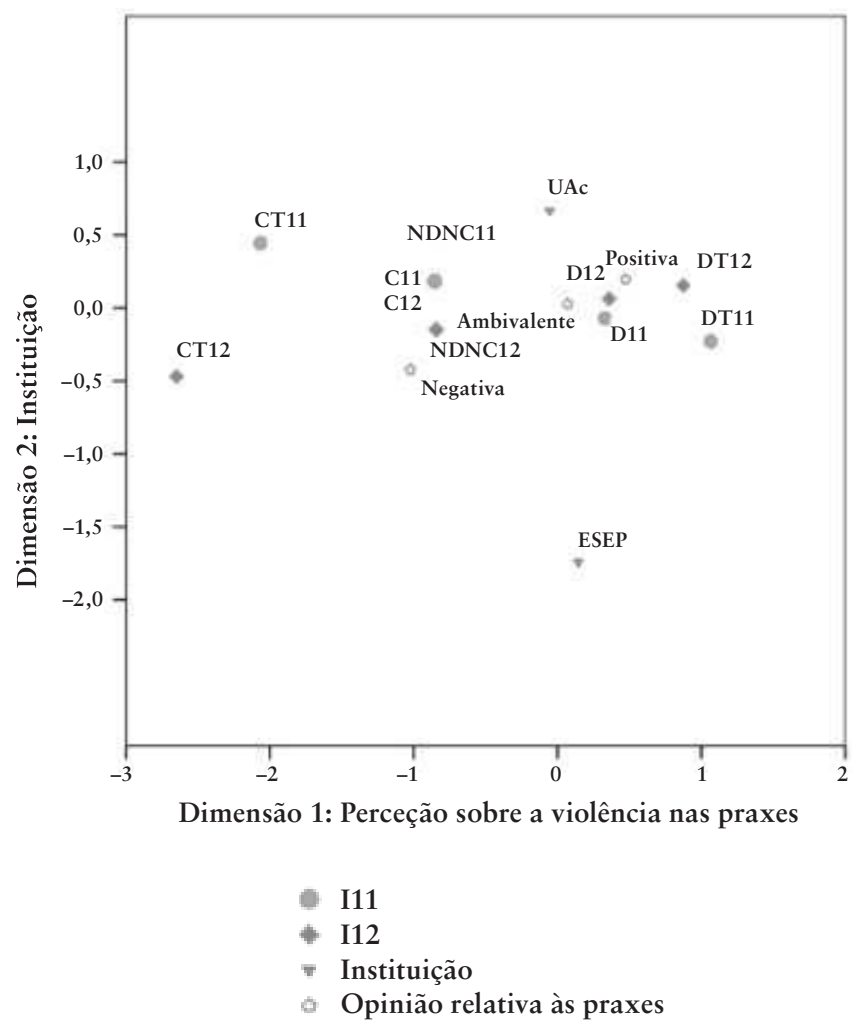

a que mais contribui para a dimensão 2 . Note-se a elevada proximidade entre as categorias "UAc" e "NDNC11", o que reforça a ideia supracitada de que, comparativamente aos estudantes da ESEP, os da UAc tendem mais a responder "Não concordo nem discordo" relativamente à afirmação referente ao item 11. É de salientar ainda que os estudantes que não concordam nem discordam com a afirmação do item 12 tendem a ter uma opinião ambivalente ou negativa relativamente às praxes. Por outro lado, os que discordam no todo ou em parte com as afirmações referentes aos itens 11 e 12 tendem a ter uma opinião/perceção positiva sobre as praxes.

Com vista à identificação de uma tipologia de variáveis relativas à EASVE, foi efetuada a ACHA, apresentando-se na Figura 5 o dendrograma obtido por todos os métodos aplicados.

De acordo com o valor (3.7321) obtido para a estatística global de níveis, STAT, a partição mais significativa contém duas classes: $C_{1}:\{V 1, V 5, V 6, V 2$, 
Figura 5.

Dendrograma obtido pelos métodos SL, AVL, AVL, AV1 e AVB.

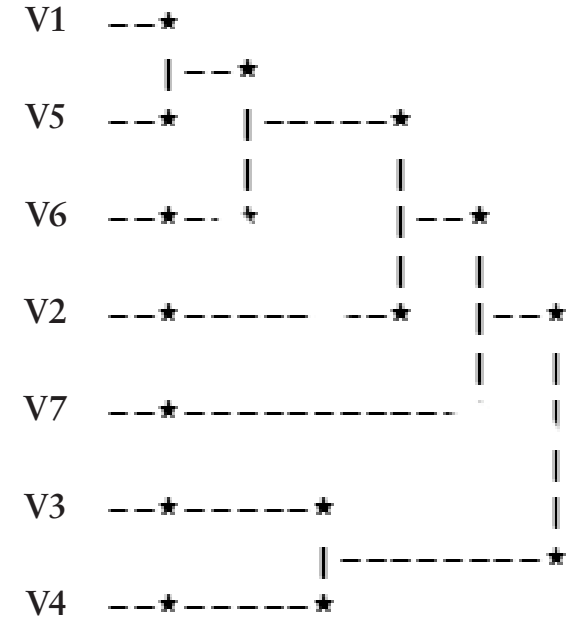

V7\}; e $\mathrm{C}_{2}$ : $\{\mathrm{V} 3, \mathrm{~V} 4\}$. É de salientar a elevada proximidade entre os itens V1 ("A minha vida está a correr bem") e V5 ("Eu tenho uma vida boa"), e entre a subclasse formada por estes dois itens e o item V6 ("Eu tenho na vida o que quero"). É também notória a elevada proximidade entre os itens V3 ("Eu gostaria de mudar muitas coisas na minha vida") e V4 ("Eu desejava ter uma vida diferente").

Em síntese, o valor obtido para o coeficiente de correlação de Spearman entre as respostas dadas aos itens 11 ("As praxes continham alguma violência") e 12 ("Eu fui agredido(a) por atos ou palavras"), da Escala de Avaliação das Situações de Bullying nas Praxes do Ensino Superior (EASBPES), mostram em geral que, quanto maior é o grau de concordância (e, respetivamente, de discordância) com a afirmação referente ao item 12, maior é o grau de concordância (e, respetivamente, de discordância) com a afirmação subjacente ao item 11. Esta constatação apoia a hipótese de que as experiências pessoais e, consequentemente, as emoções vividas durante as praxes influenciam a perceção dos estudantes relativamente às mesmas. É de salientar, ainda, a existência de associações significativas entre: a opinião relativa às praxes e o ano do curso, o item 11 e a instituição, o item 12 e a instituição. Conforme foi evidenciado pelo mapa percetual da Análise em Componentes Principais Categórica, os estudantes que não concordam nem discordam com a afirmação do item 12 tendem a ter uma opinião ambivalente ou negativa relativamente às praxes. Por outro lado, os que discordam no todo ou em parte com as afirmações referentes aos itens 11 e 12 tendem a ter uma opinião/perceção positiva sobre as praxes. 
Embora os níveis de satisfação com a vida, avaliados pelas pontuações obtidas na EASVE, não estejam fortemente correlacionadas com as perceções dos estudantes sobre a violência das praxes (itens 11 e 12), verificou-se a existência de correlações negativas muito baixas entre o item 11 e a pontuação total da EASVE, e entre o item 12 e a pontuação total da EASVE, quer considerando a amostra global, quer considerando de forma isolada os estudantes do sexo masculino, os do sexo feminino, os da Universidade dos Açores e os da Escola Superior de Enfermagem de Portalegre.

\section{Considerações finais}

Este trabalho procurou conhecer as perceções de estudantes sobre a existência ou não de violência nas praxes e relacionar essas perceções com os seus níveis de satisfação com a vida.

Foram inquiridos estudantes de duas instituições de ensino superior situadas em zonas do país relativamente afastadas dos grandes centros urbanos, nomeadamente, da Escola Superior de Enfermagem de Portalegre e da Universidade dos Açores, através de um processo de inquérito, contendo duas escalas de resposta fechada e um item onde os estudantes deveriam adjetivar livremente a praxe.

Em termos de perceção sobre a existência de violência nas praxes, os resultados globais das respostas aos itens 11 e 12 da EASBPES, cujos enunciados são, respetivamente, "As praxes continham alguma violência" e "Eu fui agredido(a) por palavras ou atos", indicam que cerca de $10 \%$ dos estudantes inquiridos perspetivaram os rituais iniciáticos como um acontecimento violento, sendo essa perceção relativamente mais expressiva em Portalegre. Neste último contexto, a sensação de abuso e subjugação indicada por alguns estudantes parece persistir no tempo, já que em 2009 aqueles haviam denunciado a violência na praxe durante um "tribunal de praxe" (Cabral e Mineiro, 2015).

Apesar de alguns estudantes perceberem a praxe como algo negativo, como se acabou de mencionar, a análise dos dois itens da EASBPES indica que a opinião predominante dos inquiridos é positiva e de diversão estudantil, sendo esta conclusão de certa forma corroborada pela expressão livre daqueles que as apelidam de "integradoras" e "divertidas". Não obstante, não é de descurar que, nessa expressão livre acerca da praxe, surge, na terceira posição, a ideia de ela ser "desnecessária". Este aspeto pode alertar para a necessidade de fortalecer a criação de alternativas no acolhimento e integração dos novos estudantes numa lógica de respeito mútuo, de equidade e paridade nas relações, e em harmonia com os princípios de uma sociedade contra-hegemónica.

No respeitante aos níveis de satisfação com a vida, avaliados pelas pontuações obtidas na EASVE, regista-se que não estão fortemente correlacionados com as perceções dos estudantes sobre a violência das praxes (itens 11 e 12 da 
EASBPES), podendo significar que este acontecimento, apesar de poder ter correspondido, para alguns estudantes, a um tempo de humilhação e violência, não constrange significativamente o sentido e o projeto de vida de cada um.

Importa, contudo, não menosprezar a influência perniciosa de alguns grupos organizados de praxistas, lembrando que têm ocorrido situações extremas que levaram à morte de estudantes. Importa igualmente reter que outros autores, do campo da sociologia e da antropologia, como por exemplo Dias e Sá (2013) ou Mendes e colaboradores (2013), utilizando outro tipo de metodologias, nomeadamente métodos qualitativos tais como entrevistas e focus grupos, também identificaram algumas manifestações de sexismo, machismo e violência nas praxes, bem como alguma ambivalência na vivência da praxe académica, e uma divisão entre os estudantes no que respeita às opiniões e modos como vivem a praxe.

Assim, apesar de os resultados obtidos nas duas instituições estudadas serem relativamente encorajadores no que respeita à forma como os estudantes se sentem acolhidos, os resultados obtidos, ainda indiciadores de alguma existência de práticas ofensivas, sugerem que o estudo da transição do ensino secundário para o superior, do fenómeno da vivência da praxe e da ativação de alternativas à praxe carecem de maior atenção e mais investigação.

\section{Referências bibliográficas}

BACELAR-NICOLAU, H. (1980), "Contributions to the study of comparison coefficients in cluster analysis”, Dissertação de Doutoramento (in Portuguese). Lisboa: Universidade de Lisboa.

BACELAR-NICOLAU, H. (1985), "The affinity coefficient in cluster analysis", Methods of Operations Research, vol. 53, M. J. Beckmann, K.-W. Gaede, K. Ritter, \& H. Schneeweiss (Eds.), Verlag Anton Hain, Munchen: 507-512.

BACELAR-NICOLAU, H. (1988), "Two probabilistic models for classification of variables in frequency tables", in H.-H. Bock, Classification and Related Methods of Data Analysis Ed. North Holland: Elsevier Sciences Publishers B.V.: 181-186.

CABRAL, B. M. \& MINEIRO, J. (2015), Desobedecer à Praxe. Porto: Deriva Editores.

CALDEIRA, S. N.; SILVA, O.; MENDES, M., \& BOTELHO, S. (2015), "Hazing Practices in Higher Education: a study with Portuguese students". International Journal of Current Research, 7(04): 15444-15447.

COCCO M.; LOPES M. J. M. (2010), "Violência entre jovens: dinâmicas sociais e situações de vulnerabilidade". Revista Gaúcha Enfermagem, 31(1): 151-159.

DIÁRIO DE NOTÍCIAS (15 de dezembro de 2013), Sete jovens desaparecidos na praia do Meco. Acedido a 27 de junho de 2015 em http://www.dn.pt/inicio/portugal/interior. aspx?content_id=3588892.

DIAS, D. \& SÁ, M. (2013), "Rituais de transição no ensino superior português: A praxe enquanto processo de reconfiguração identitária”. Revista Galego-Portuguesa de Psicoloxía e Educación, 21(1). Disponível em http://ruc.udc.es/bitstream/2183/12618/1/RGP_21_2013_art_2.pdf.

FERRO, J. P. (2013), Violência escolar em foco: percepções e encaminhamentos de professores e gestores, Dissertação de Mestrado apresentada ao Programa de Pós-Graduação em Educação. Paranaíba: Universidade Estadual de Mato Grosso do Sul.

LERMAN, I.C. (1981), Classification et analyse ordinale des données. Paris: Dunod. 
LERMAN, I.C. (1972), Étude distributionnelle de statistiques de proximité entre structures algébriques finies du même type - Application à la Classification Automatique, Cahiers du B.U.R.O., Paris, No. 19, 1972.

MARQUES, S. \& PAIS RIBEIRO, J. L. (2006), “Contribuição para o estudo psicométrico e estrutural da escala abreviada de satisfação com a vida para estudantes: estudo preliminar", in C. Machado, C. Almeida, M. A. Guisande, M. Gonçalves \& V. Ramalho (Eds.), Actas da XI Conferência Internacional de Avaliação Psicológica: Formas e contextos. Lisboa: Edições Psiquilibrios: 536-543.

MARTINS, M. J. D. (2007), "Violência interpessoal e maus-tratos entre pares, em contexto escolar”. Revista de Educação, XV, 2: 51-78, disponível em http://revista.educ.fc.ul.pt/.

MASCARENHAS, S.; MATOS, F.; JESUS, S., \& GALDINO, Z. (n.d.), Diagnóstico e avaliação das praxes ou trotes - Um estudo transcultural com universitários de Portugal e do Brasil. Acedido em abril 2014: http://portal.metodista.br/metodista.br/ev/psicologia-da-saude/ anais1/2011/comunicacaooral/co22/TEXTO $\% 20$ INTEGRAL\%20\%20Escala\%20Para $\% 20$ Avaliacao\%20Das\%20Situacoes\%20De\%20Bullying \%20Nos\%20Trotes\%20Do\% 20 Ensino\%20Superior\%20Ap.pdf/view.

MENDES, J. M.; DUARTE, M.; ARAÚJO, P., \& LOPES, R. (2013), "Violência e relações de intimidade no ensino superior em Portugal: representações e práticas". Teoria e Sociedade, 21 (2): 87-112.

MYSYUK, Y.; WESTENDORPA, R. G. J. \& LINDENBERG, J. (2013), "Added value of elder abuse definitions: A review". Ageing Research Reviews, 12(1): 50-57.

NICOLAU, F. C. \& H. BACELAR-NICOLAU, H. (1998), "Some trends in the classification of variables”, in Hayashi et al., Data Science, Classification and Related Methods. Ed. Springer: 89-98.

NICOLAU, F. C. (1983), "Cluster analysis and distribution function", Methods of Operations Research, vol. 45: 431-433.

NIRH, J. (2014), Explanations of college students for engaging in hazing. Dissertação para o grau de Doutor em Filosofia com especialização em Ensino Superior. Arizona: Universidade do Arizona.

PIMENTEL, M.; MATA, M. \& PEREIRA, F. (2012), "Práticas iniciáticas de integração no ensino superior. Um ritual institucionalizado ou um processo de (des)integração?", in Atas do V Encontro do CIED - Escola e Comunidade. Lisboa: Escola Superior de Educação de Lisboa: 393-401.

RUOTTI, C. (2010), "Violência em meio escolar: fatos e representações na produção da realidade”, Educação e Pesquisa, São Paulo, 36(1): 339-355, disponível em http://www.scielo.br/ $\mathrm{pdf} / \mathrm{ep} / \mathrm{v} 36 \mathrm{n} 1 / \mathrm{a} 10 \mathrm{v} 36 \mathrm{n} 1 . \mathrm{pdf}$

SILVA, O.; CALDEIRA, S. N.; MENDES, M. \& BOTELHO, S. (2014), "Bullying in hazing practices: a study on higher education". In Proceeedings do 7 th International Conference of Education, Research and Innovation. ICERI, Seville, Spain, 17-19 de novembro 2014: 784-791.

V PLANO NACIONAL DE PREVENÇÃO E COMBATE À VIOLÊNCIA DOMÉSTICA E DE GÉNERO, 2014-2017, disponível em http://www.portugal.gov.pt/media/1250632/V\%20 PNPCVDG\%20\%20Consulta\%20publica.pdf.

VIEIRA, P. (2013), Vivências da praxe académica: percepção de integração e ansiedade na transição para o ensino superior. Dissertação de Mestrado em Psicologia da Educação, Desenvolvimento e Aconselhamento. Coimbra: Universidade de Coimbra.

WORLD HEALTH ORGANIZATION. (2002), Toronto Declaration on the Global Prevention of Elderly Abuse, disponível em http://www.who.int/ageing/projects/elder_abuse/ alc_toronto_declaration_en.pdf.

ZANATTA, E. A. (2013). Compreensão de jovens universitários sobre a violência - Sob o olhar da corporeidade, da vulnerabilidade e do cuidado. Tese de Pós-Graduação em Enfermagem. Porto Alegre, Universidade Federal de Rio Grande do Sul, disponível em http://www.lume. ufrgs.br/bitstream/handle/10183/87247/000911360.pdf?sequence=1. 